\title{
STANDARD BASES FOR THE UNIVERSAL ASSOCIATIVE CONFORMAL ENVELOPES OF KAC-MOODY CONFORMAL ALGEBRAS
}

\author{
P.S. KOLESNIKOV ${ }^{1)}$, R.A. KOZLOV ${ }^{1) 2}$
}

\begin{abstract}
We study the universal enveloping associative conformal algebra for the central extension of a current Lie conformal algebra at the locality level $N=3$. A standard basis of defining relations for this algebra is explicitly calculated. As a corollary, we find a linear basis of the free commutative conformal algebra relative to the locality $N=3$ on the generators.
\end{abstract}

\section{INTRODUCTION}

Conformal algebras also known as Lie vertex algebras were introduced in [16 as an algebraic tool to study the singular part of the operator product expansion (OPE) of chiral fields in 2-dimensional conformal field theory coming back to [5. From the categorical point of view, a conformal algebra is just an algebra in the appropriate (pseudo-tensor) category $\mathcal{M}^{*}(\mathbb{C}[\partial])$ of modules over the polynomial algebra $\mathbb{C}[\partial]$ in one variable [2]. The pseudo-tensor structure (see [4]) reflects the main features of multi-linear maps in the category of linear spaces: composition, identity, symmetric structure. These features are enough to define the basic notions like what is an algebra (associative, commutative, Lie, etc.), homomorphism, ideal, representation, module, cohomology. Therefore, the notion of a conformal algebra is a natural expansion of the notion of an "ordinary" algebra over $\mathbb{C}$ to the pseudo-tensor category $\mathcal{M}^{*}(\mathbb{C}[\partial])$. Namely, as an ordinary algebra is a linear space equipped with a bilinear product, a conformal algebra is a $\mathbb{C}[\partial]$-module $V$ equipped with a $\mathbb{C}[\partial]$-bilinear map (pseudo-product)

$$
*: V \otimes V \rightarrow \mathbb{C}[\partial]^{\otimes 2} \otimes_{\mathbb{C}[\partial]} V .
$$

A more convenient presentation for the operation $*$ uses the language of a $\lambda$-product or a family of $n$-products for all integer $n \geq 0$ ([16], see also Section 2).

Conformal algebras representing the singular part of OPE in vertex algebras are Lie algebras in the category $\mathcal{M}^{*}(\mathbb{C}[\partial])$, i.e., Lie conformal algebras. For example, if $\mathfrak{g}$ is a Lie algebra then the free module $\mathbb{C}[\partial] \otimes \mathfrak{g}$ equipped with the pseudo-product $a * b=(1 \otimes 1) \otimes_{\mathbb{C}[\partial]}[a, b], a, b \in \mathfrak{g}$, is a Lie conformal algebra denoted Cur $\mathfrak{g}$ (current

1991 Mathematics Subject Classification. 17A61, 17B35, 17B69 .

Key words and phrases. conformal algebra, Gröbner-Shirshov basis.

The work is supported by Mathematical Center in Akademgorodok. 
conformal algebra). If $\langle\cdot \mid \cdot\rangle$ is a bilinear symmetric invariant form on $\mathfrak{g}$ then Cur $\mathfrak{g}$ has a 1-dimensional central extension $K(\mathfrak{g})$ defined by

$$
a * b=(1 \otimes 1) \otimes_{\mathbb{C}[\partial]}[a, b]-(\partial \otimes 1) \otimes_{\mathbb{C}[\partial]}\langle a \mid b\rangle e,
$$

where $e$ is a central element and $\partial e=0$. For example, in the Kac-Moody vertex algebra $V(\mathfrak{g})$ [12] the singular part of the OPE on the generating fields is described by this particular structure $K(\mathfrak{g})$ called a Kac-Moody conformal algebra.

As in the case of ordinary algebras, an associative conformal algebra $C$ turns into a Lie one with respect to the commutator $[a * b]=(a * b)-\left(\tau \otimes_{\mathbb{C}[\partial]} 1\right)(b * a), a, b \in C$, where $\tau$ is the switching map on $\mathbb{C}[\partial]^{\otimes 2}$. However, not all Lie conformal algebras embeds into associative ones in this way [26]. This is an open problem whether every finite (i.e., finitely generated as a $\mathbb{C}[\partial]$-module) Lie conformal algebra embeds into an associative conformal algebra with respect to conformal commutator. Even for the class of quadratic conformal algebras [27] (see also [15]) it remains unknown in general if every such Lie conformal algebra embeds into an appropriate associative one.

A routine way to solve this kind of problems is to construct a universal envelope. In general, such an algebra is defined by generators and relations. For a Lie conformal algebra, there exists a lattice of universal enveloping associative conformal algebras, each related to an (associative) locality bound on the generators ([26], see also Section 3.4). In order to prove (or disprove) the embedding of a Lie conformal algebra into its universal enveloping associative conformal algebra one needs to know the normal form of elements in the last algebra.

A general and powerful method for finding normal forms in an algebra defined by generators and relations is to calculate a standard (or Gröbner-Shirshov) basis of defining relations. The idea goes back to Newmann's Diamond Lemma [23], see also [7, 6]. In the recent years, the Gröbner-Shirshov bases theory was developed to serve the problem of combinatorial analysis of various algebraic structures, see [9]. For associative conformal algebras it was initially invented in [8], later developed in [24] and [20]. In this paper, we use the last approach exposed in a form convenient for actual computation: we consider defining relations in a conformal algebra as rewriting rules on a module over an appropriate associative algebra (the GröbnerShirshov basis of the last algebra is known).

A series of particular observations made in [21], 22] shows that for all considered examples of quadratic Lie conformal algebras $L$ it is enough to consider universal associative conformal envelopes $U$ relative to the locality bound $N=3$ to get an injective mapping $L \curvearrowright U$. This is one of the reasons why we focus on the locality bound $N=3$ for the envelopes of current conformal algebras as they are particular examples of quadratic conformal algebras.

The main purpose of this paper is to find a standard (Gröbner-Shirshov) basis of defining relations for the universal enveloping associative conformal algebra of a Kac-Moody conformal algebra at locality level $N=3$. As a corollary, we get an analogue of the Poincaré-Birkhoff-Witt Theorem (PBW-Theorem) stating that 
the associated graded conformal algebra obtained from the universal envelope of a current Lie conformal algebra with respect to the natural filtration is isomorphic to the free commutative conformal algebra. Note that the classical PBW-Theorem may be interpreted as a conformal one at the locality level $N=1$ : for a Lie algebra $\mathfrak{g}$, its "ordinary" universal envelope $U(\mathfrak{g})$ gives rise to the conformal algebra Cur $U(\mathfrak{g})_{0}$ which is exactly the universal enveloping conformal algebra of Cur $\mathfrak{g}$ with $N=1$ (here $U(\mathfrak{g})_{0}$ is the augmentation ideal of $\left.U(\mathfrak{g})\right)$.

There are several reasons for studying the universal envelopes of Cur $\mathfrak{g}$ at higher locality than $N=1$.

First, the $N=1$ envelope $\operatorname{Cur} U(\mathfrak{g})$ does not reflect the homological properties of Cur $\mathfrak{g}$. For example, if $\mathfrak{g}$ is a simple finite-dimensional Lie algebra then the second cohomology group $H^{2}(\operatorname{Cur} \mathfrak{g}, \mathbb{k})$ is one-dimensional [3]. The corresponding central extension is the Kac-Moody conformal algebra $K(\mathfrak{g})$ representing the singular part of the Kac-Moody vertex algebra [12]. On the other hand, it is easy to find that the second Hochschild cohomology group of Cur $U(\mathfrak{g})_{0}$ with coefficients in the trivial 1-dimensional module is zero: there are no nontrivial central extensions. Our results show that the universal enveloping associative conformal algebras for Cur $\mathfrak{g}$ at locality level $N=2,3$ do have a nontrivial central extension which is exactly the universal envelope of $K(\mathfrak{g})$.

The second reason is related with Poisson algebras. Assume $P$ is an ordinary commutative algebra with a Poisson bracket $\{\cdot, \cdot\}$. Then Cur $P$ may be considered as a Lie conformal algebra since $P$ is a Lie algebra relative to the Poisson bracket. There is a conformal representation of $\operatorname{Cur} P$ on itself given by the rule

$$
\left(a_{(\lambda)} f\right)=\{a, f\}+\lambda a f, \quad a, f \in P .
$$

The study of this representation provides a way to get new results in (quadratic) conformal algebras as well as in Poisson algebras [21, 22]. The conformal linear operators $\rho(a) \in \operatorname{Cend}(\operatorname{Cur} P), \rho\left(a_{\lambda}: f \mapsto\left(a_{(\lambda)} f\right)\right.$, are local to each other, and the locality bound is $N=3$. Indeed, according to the definition of a conformal representation [11, 16] we have

$$
\begin{aligned}
\left(\rho(a)_{(\lambda)} \rho(b)\right)_{\mu} f=a_{(\lambda)} & \left(b_{(\mu-\lambda)} f\right) \\
= & \{a,\{b, f\}\}+\lambda a\{b, f\}+(\mu-\lambda)\{a, b f\}+\lambda(\mu-\lambda) a b f,
\end{aligned}
$$

for $a, b, f \in P$. If $a b f \neq 0$ then right-hand side is of degree 2 in $\lambda$ that means $N(\rho(a), \rho(b))=3$ in Cend(Cur $P$ ). Therefore, the corresponding associative envelope belongs to the class of envelopes with locality $N=3$.

The third reason to study the case $N=3$ comes from the following relation between commutative conformal and Novikov algebras. Suppose $C$ is a commutative conformal algebra and $M$ is a subset of $C$ such that $N_{C}(a, b) \leq 3$ for all $a, b \in M$. Then $M$ generates an ordinary (nonassociative) subalgebra $N(M)$ in the space $C$ considered relative to the single product $x \circ y=x_{(1)} y$. Indeed, all elements of $N(M)$ 
are local to each other with locality bound 3. Moreover, the following relations hold:

$$
\begin{aligned}
(x \circ y) \circ z-x \circ(y \circ z) & =(x \circ z) \circ y-x \circ(z \circ y), \\
x \circ(y \circ z) & =y \circ(x \circ z),
\end{aligned}
$$

for all $x, y, z \in N(M)$. These identities are known to define the variety of Novikov algebras initially appeared in [1], [13]. In order to perform a systematic study of this relation, one needs to know the structure of the universal object in the category of commutative conformal algebras with locality bound $N=3$ on the generators.

For all these reasons, we study the universal enveloping associative conformal algebras for Kac-Moody conformal algebras $K(\mathfrak{g})$ relative to the locality level $N=3$. The corollaries of the main result of the paper (Theorem 2) allow us to get the structure of the universal envelopes for current Lie conformal conformal algebras at $N=3$ and also describe the free commutative conformal algebra at the same locality level. Practically, we find a standard (Gröbner-Shirshov) basis of defining relations for these conformal algebras and derive an analogue of the PBW-Theorem.

\section{Preliminaries in CONFORMal ALGEBras}

The definition of a conformal algebra as an algebra in an appropriate pseudotensor category [2] corresponds to the convenient algebraic approach using $\lambda$-brackets [16] if it is presented in terms of operads associated with linear algebraic groups [18].

Let $G$ be a linear algebraic group over a field $\mathbb{k}$ of characteristic zero, and let $H_{G}=\mathbb{k}[G]$ be the Hopf algebra of regular functions on $G$. For every $H_{G}$-module $V$ there is a non-symmetric operad (let us denote it $V_{G}$ ) defined as follows. Given $n \in\{1,2, \ldots\}$, set

$$
V_{G}(n)=\left\{f: G^{n-1} \rightarrow \operatorname{Hom}\left(V^{\otimes n}, V\right) \mid f \text { is regular and 3/2-linear }\right\}
$$

The condition of regularity means that $f$ may be presented by a polynomial function with coefficients in the space $\operatorname{Hom}\left(V^{\otimes n}, V\right)$ of $\mathbb{k}$-polylinear maps on $V$, and the $3 / 2$ linearity (sesqui-linearity) may be expressed as

$$
f\left(\lambda_{1}, \ldots, \lambda_{n-1}\right):\left(v_{1}, \ldots, h(x) v_{i}, \ldots, v_{n}\right) \mapsto \begin{cases}h\left(\lambda_{i}^{-1}\right) v, & i=1, \ldots, n-1, \\ h\left(\lambda_{n-1} \ldots \lambda_{1} x\right) v, & i=n\end{cases}
$$

for $v=f\left(\lambda_{1}, \ldots, \lambda_{n-1}\right)\left(v_{1}, \ldots, v_{n}\right), \lambda_{i} \in G, v_{i} \in V, h(x) \in H_{G}$ (here $x$ is a variable ranging in $G$ ). In particular, $V_{G}(1)$ is the space of all $H_{G}$-linear transformations of $V$ thus contains the identity map id.

The composition rule in $V_{G}$ is defined by the following partial composition. If $f \in V_{G}(n), g \in V_{G}(m), i \in\{1, \ldots, n\}$ then

$$
f \circ_{i} g=f\left(\mathrm{id}, \ldots, g_{i}, \ldots, \mathrm{id}\right) \in V_{G}(n+m-1)
$$


acts as

$$
\begin{aligned}
& f \circ_{i} g:\left(\lambda_{1}, \ldots, \lambda_{i-1}, \mu_{1}, \ldots, \mu_{m-1}, \lambda_{i}, \ldots, \lambda_{n-1}\right) \\
& \mapsto f\left(\lambda_{1}, \ldots, \lambda_{i-1}, \lambda_{i} \mu_{m-1} \ldots \mu_{1}, \lambda_{i+1}, \ldots, \lambda_{n-1}\right) \circ_{i} g\left(\mu_{1}, \ldots, \mu_{m-1}\right),
\end{aligned}
$$

for $\lambda_{i}, \mu_{j} \in G$, where $o_{i}$ in the right-hand side stands for the ordinary partial composition of polylinear maps. In particular, for $i=n$ the partial composition is equal to

$$
f\left(\lambda_{1}, \ldots, \lambda_{n-1}\right) \circ_{n} g\left(\mu_{1}, \ldots, \mu_{m-1}\right) .
$$

It is easy to see that the resulting maps are indeed regular and $3 / 2$-linear.

One may easily check that the partial composition in $V_{G}$ defined above meets the sequental, parallel, and unit axioms [10, Definition 3.2.2.3] and thus this is indeed an non-symmetric operad with a well-defined composition rule

$$
\gamma_{m_{1}, \ldots, m_{r}}^{r}: V_{G}(r) \otimes V_{G}\left(m_{1}\right) \otimes \cdots \otimes V_{G}\left(m_{r}\right) \rightarrow V_{G}\left(m_{1}+\cdots+m_{r}\right) .
$$

Suppose the group $G$ is abelian. Then $V_{G}(n)$ has a natural action of the symmetric group $S_{n}$ defined in the following way. If $f \in V_{G}(n)$ and $(1 i), i=2, \ldots, n$, is a transposition in $S_{n}$ then

$$
f^{(1 i)}\left(\lambda_{1}, \ldots, \lambda_{n-1}\right)=f\left(\lambda_{i}, \lambda_{2}, \ldots, \lambda_{i}, \ldots \lambda_{n-1}\right)^{(1 i)}
$$

for $i<n$ (here the action of $(1 i)$ in the right-hand side is just the permutation of arguments in a polylinear map). For $i=n$, the definition is slightly more complicated: if $f$ is presented by a polynomial function

$$
f=\sum_{i} f_{i}\left(x_{1}, \ldots, x_{n-1}\right) \varphi_{i}, \quad f_{i} \in H_{G}^{\otimes n-1} \simeq H_{G^{n-1}}, \varphi_{i} \in \operatorname{Hom}\left(V^{\otimes n}, V\right)
$$

then $f^{(1 n)}\left(\lambda_{1}, \ldots, \lambda_{n-1}\right)$ is given by

$$
\sum_{i} f_{i}^{\prime}(x) \varphi_{i}^{(1 n)}
$$

where the each $f_{i}^{\prime}(x) \in H_{G}$ is the regular function $f_{i}\left(\left(\lambda_{1} \ldots \lambda_{n-1} x\right)^{-1}, \lambda_{2}, \ldots, \lambda_{n-1}\right)$.

The composition rule $\gamma_{m_{1}, \ldots, m_{r}}^{r}$ is equivariant (see, e.g., [10, Definition 5.2.1.1]) since the structure obtained is equivalent to the structure of an $H_{G}$-module operad defined over a cocommutative Hopf algebra [2]. Namely, one may identify a map

$$
F: V^{\otimes n} \rightarrow H_{G}^{\otimes n} \otimes_{H_{G}} V \simeq H_{G}^{\otimes n-1} \otimes V \simeq H_{G^{n-1}} \otimes V,
$$

with

$$
f\left(\lambda_{1}, \ldots, \lambda_{n-1}\right)=F\left(\lambda_{1}^{-1}, \ldots, \lambda_{n}^{-1}\right) .
$$

Recall that if $\mathcal{O}$ is a (symmetric) operad then a morphism $\mathcal{O} \rightarrow V_{G}$ defines an algebraic structure on the space $V$. In the trivial case $G=\{e\}, H_{G}=\mathbb{k}$, the operad $V_{G}$ coincides with the operad of polylinear maps on the linear space $V$, and thus a morphism $\mathcal{O} \rightarrow V_{G}$ defines the ordinary notion of an $\mathcal{O}$-algebra over $\mathbb{k}$, a space equipped with a family of polylinear operations. 
The classes of conformal [16] and $\mathbb{Z}$-conformal [14] algebras naturally appear in the next step, if we choose $G$ to be a connected linear algebraic group of dimension 1 (the affine line and $G L_{1}$, respectively). For a non-connected group $G$ with the identity component denoted $G^{0}$, the structure of a conformal algebra over $G$ is naturally interpreted as a $G / G^{0}$-graded conformal algebra over $G^{0}[19]$. If $G=\mathbb{A}_{1}=(\mathbb{k},+)$, $H_{G}=\mathbb{k}[\partial]$ (the variable is traditionally denoted by $\partial$ ), then a morphism $\mathcal{O} \rightarrow V_{G}$ defines a $\mathcal{O}$-conformal algebra structure on a $\mathbb{k}[\partial]$-module $V$.

For example, if $\mathcal{O}=$ As is the operad governing the variety of associative algebras (generated by $\mu=x_{1} x_{2} \in \operatorname{As}(2)$ modulo the relation $\mu \circ_{1} \mu=\mu \circ_{2} \mu$ ) then an associative conformal algebra structure on a $\mathbb{k}[\partial]$-module $V$ is given by an image of $\mu$, a map $f=\left(\cdot{ }_{(\lambda)} \cdot\right): V \otimes V \rightarrow \mathbb{k}[\lambda] \otimes V$ which is 3/2-linear

$$
\left(\partial v_{(\lambda)} u\right)=-\lambda\left(v_{(\lambda)} u\right), \quad\left(v_{(\lambda)} \partial u\right)=(\partial+\lambda)\left(v_{(\lambda)} u\right),
$$

for $u, v \in V$, and associative in the sense that

$$
\left(f \circ_{2} f\right)(\lambda, \mu)=\left(f \circ_{1} f\right)(\lambda, \mu) .
$$

By (11), the latter means

$$
\left(u_{(\lambda)}\left(v_{(\mu)} w\right)\right)=\left(\left(u_{(\lambda)} v\right)_{(\lambda+\mu)} w\right)
$$

(to compute the right-hand side, put $\mu_{1}=\lambda$ and $\lambda_{1}=\mu$ in (10)).

According to the same scheme, a Lie conformal algebra structure on a $\mathbb{k}[\partial]$-module $V$ is a morphism from the operad Lie governing the variety of Lie algebras to $V_{\mathbb{A}_{1}}$. To define such a morphism, it is enough to fix a 3/2-linear map $\mu \in V_{\mathbb{A}_{1}}(2)$

$$
\mu=\left[\cdot{ }_{(\lambda)} \cdot\right]: V \otimes V \rightarrow \mathbb{k}[\lambda] \otimes V
$$

such that $\mu^{(12)}=-\mu$ and $\left(\mu \circ_{2} \mu\right)-\left(\mu \circ_{2} \mu\right)^{(12)}=\left(\mu \circ_{1} \mu\right)$. The last two relations represent anti-commutativity and Jacobi identity, respectively:

$$
\begin{gathered}
{\left[u_{(-\partial-\lambda)} v\right]=-\left[v_{(\lambda)} u\right]} \\
\left.\left[u_{(\lambda)}\left[v_{(\mu)} w\right]\right]-\left[v_{(\mu)}\left[u_{(\lambda)} w\right]\right]=\left[u_{(\lambda)} v\right]_{(\lambda+\mu)} w\right]
\end{gathered}
$$

$u, v, w \in V$.

In the sequel, we will use the notation $(\cdot(\lambda) \cdot)$ for the operation on an associative conformal algebra and $[\cdot(\lambda) \cdot]$ for Lie conformal algebras.

Since there is a morphism of operads $(-):$ Lie $\rightarrow$ As sending $\mu$ to $f-f^{(12)}$, every associative conformal algebra turns into a Lie conformal algebra relative to the operation

$$
\left[u_{(\lambda)} v\right]=\left(u_{(\lambda)} v\right)-\left(v_{(-\partial-\lambda)} u\right) .
$$

For an associative conformal algebra $V$ defined via a morphism of operads As $\rightarrow V_{\mathbb{A}_{1}}$, let $V^{(-)}$stand for the Lie conformal algebra obtained as a composition Lie $\stackrel{(-)}{\rightarrow} \mathrm{As} \rightarrow$ $V_{\mathbb{A}_{1}}$. 
The property of a commutator to be a derivation on an associative algebra may also be expressed as a relation in As(3). Being translated to conformal algebras it turns into the following identity on an associative conformal algebra $V$ :

$$
\left(u_{(\lambda)}\left(v_{(\mu)} w\right)\right)-\left(v_{(\mu)}\left(u_{(\lambda)} w\right)\right)=\left(\left[u_{(\lambda)} v\right]_{(\lambda+\mu)} w\right), \quad u, v, w \in V .
$$

As in the case of ordinary algebras, $V \mapsto V^{(-)}$is a functor from the category of associative conformal algebras to the category of Lie algebras. In contrast to the case of ordinary algebras, this functor does not have a left adjoint one when considered on the entire category of associative conformal algebras. However, if we restrict the class of associative conformal algebras by means of locality on the generators ([26], see Section 3.4 for details) then there is an analogue of the universal enveloping associative algebra for Lie conformal algebras.

In terms of "ordinary" algebraic operations, a conformal algebra is a linear space $V$ equipped with a linear operator $\partial$, the generator of $H_{\mathbb{A}_{1}}=\mathbb{k}[\partial]$, and a series of bilinear operations $\left(\cdot{ }_{(n)} \cdot\right), n \in \mathbb{Z}_{+}$, given by

$$
\left(u_{(\lambda)} v\right)=\sum_{n \geq 0} \frac{\lambda^{n}}{n !}\left(u_{(n)} v\right), \quad u, v \in V .
$$

These operations are called $n$-products. They have to satisfy the following properties:

(C1) For every $u, v \in V$ there exists $N=N(u, v)$ such that $\left(u_{(n)} v\right)=0$ for all $n \geq N$

(C2) $\left(\partial u_{(n)} v\right)=-n\left(u_{(n-1)} v\right)$;

(C3) $\left(u_{(n)} \partial v\right)=\partial\left(u_{(n)} v\right)+n\left(u_{(n-1)} v\right)$.

The property $(\mathrm{C} 1)$ is known as the locality axiom, (C2) and (C3) represent 3/2linearity. For every conformal algebra $V$, the locality function $N_{V}$ is a map $V \times V \rightarrow$ $\mathbb{Z}_{+}$such that $u_{(n)} v=0$ for every $u, v \in V$ and $n \geq N_{V}(u, v)$.

A conformal algebra $V$ is associative if

$$
\left(u_{(n)}\left(v_{(m)} w\right)\right)=\sum_{s=0}^{n}\left(\begin{array}{l}
n \\
s
\end{array}\right)\left(\left(u_{(n-s)} v\right)_{(m+s)} w\right)
$$

for all $u, v, w \in V$ and $n, m \in \mathbb{Z}_{+}$. In a similar way, one may rewrite the identities defining the class of Lie conformal algebras.

Given a set $X$ and a function $N: X \times X \rightarrow \mathbb{Z}_{+}$, there exists a unique (up to isomorphism) associative conformal algebra denoted $\operatorname{Conf}(X, N)$ which is universal among all associative conformal algebras $V$ generated by $X$ such that $N_{V}(x, y) \leq$ $N(x, y)$ for all $x, y \in X$ [25]. The details of the construction of $\operatorname{Conf}(X, N)$ are stated in Section 3.3 . 


\section{GRÖBNER-SHIRShOV BASES FOR ASSOCIATIVE CONFORMAL ALGEBRAS}

3.1. Rewriting system and standard bases for associative algebras. In this section, we briefly describe the well-known technique of standard bases (GröbnerShirshov bases) in associative algebras in order to fix the notations. The usual exposition of this technique requires a proper ordering of the monomials. However, the core statements laying in the foundation of the approach do not need a monomial ordering.

Let $B$ be a set and let $B^{*}$ stand for the set of all words in $B$ (including the empty word). The free associative algebra (with a unit) over the field $\mathbb{k}$ generated by $B$ is denoted $\mathbb{k}\langle B\rangle$. Suppose $\Sigma$ is a family of pairs $(u, f)$ called rewriting rules, where $u \in B^{*}, f \in \mathbb{k}\langle B\rangle$. We will write a pair like this as $(u \rightarrow f)$ since the family $\Sigma$ determines an oriented graph $\mathcal{G}(B, \Sigma)$ as follows. The vertices of $\mathcal{G}(B, \Sigma)$ are the elements of $\mathbb{k}\langle B\rangle$; two vertices $g$ and $h$ are connected with an edge $(g \rightarrow h)$ if and only if there is a rewriting rule $u \rightarrow f$ in $\Sigma$ and a summand of the form $\alpha w$ in $g$ $\left(\alpha \in \mathbb{k}^{\times}, w \in B^{*}\right)$ such that

$$
w=v_{1} u v_{2}, \quad h=g-\alpha v_{1}(u-f) v_{2}
$$

for some $v_{1}, v_{2} \in B^{*}$. In other words, $h$ is obtained from $g$ by replacing an occurrence of the subword $u$ with the polynomial $f$.

The graph $\mathcal{G}(B, \Sigma)$ splits into connected components (in the non-oriented sense) which explicitly correspond to the elements of the quotient $\mathbb{k}\langle B \mid \Sigma\rangle=\mathbb{k}\langle B\rangle /(\Sigma)$, where $(\Sigma)$ stands for the ideal in $\mathbb{k}\langle B\rangle$ generated by all $u-f$ for $(u \rightarrow f) \in \Sigma$. In some cases, there is a way to check algorithmically whether two vertices $g, h \in \mathbb{k}\langle B\rangle$ belong to the same connected component of $\mathcal{G}(B, \Sigma)$, i.e., if the images of $f$ and $g$ are equal in $\mathbb{k}\langle B \mid \Sigma\rangle$.

An oriented graph is called a rewriting system if there are no infinite oriented paths (in particular, no oriented cycles). In a rewriting system, for every vertex $g$ there is a nonempty set $T(g)$ of terminal vertices $t$ attached to $g$, i.e., such that there is a path $g \rightarrow \cdots \rightarrow t$, but there are no edges originated at $t$. A rewriting system is confluent if for every vertex $g$ the set $T(g)$ contains a single vertex.

Definition 1. A family of rewriting rules $\Sigma$ in the free associative algebra $\mathbb{k}\langle B\rangle$ is a standard basis (Gröbner-Shirshov basis, GSB) if $\mathcal{G}(B, \Sigma)$ is a confluent rewriting system.

Obviously, if $\Sigma$ is a GSB then every connected component of $\mathcal{G}(B, \Sigma)$ has a unique terminal vertex which is a linear combination of terminal (reduced) words. This combination is called a normal form of an element in $\mathbb{k}\langle B \mid \Sigma\rangle$ : two polynomials $g$ and $h$ in $\mathbb{k}\langle B\rangle$ represent the same element of $\mathbb{k}\langle B \mid \Sigma\rangle$ if and only if their normal forms coincide. Therefore, the images of terminal words form a linear basis of $\mathbb{k}\langle B \mid \Sigma\rangle$.

The most natural way to guarantee that $\mathcal{G}(B, \Sigma)$ is a rewriting system is to make the set $B^{*}$ well-ordered relative to an order $\leq$ such that $u \leq v$ implies $w u \leq w v$ 
and $u w \leq v w$ for all $u, v, w \in B^{*}$ (i.e., $\leq$ is a monomial order), and $u>f$ for all $(u \rightarrow f) \in \Sigma$ (i.e., $u$ is greater than every monomial in $f$ ).

To check the confluence of a rewriting system $\mathcal{G}(B, \Sigma)$ one may apply the Diamond Lemma originated to [23]. The latter states that a rewriting system is confluent if an only if for every "fork" (a pair of edges $w \rightarrow g_{1}, w \rightarrow g_{2}$ ) there exist a vertex $h$ and two oriented paths $g_{1} \rightarrow \cdots \rightarrow h, g_{2} \rightarrow \cdots \rightarrow h$. If the rewriting system is $\mathcal{G}(B, \Sigma)$ then it is enough to check the Diamond condition for the following two kinds of forks:

(1) For $u_{1} \rightarrow f_{1}, u_{2} \rightarrow f_{2}$ in $\Sigma, u_{1}=v_{1} u_{2} v_{2}$, consider $w=u_{1}, g_{1}=f_{1}$, and $g_{2}=v_{1} f_{2} v_{2}$

(2) For $u_{1} \rightarrow f_{1}, u_{2} \rightarrow f_{2}$ in $\Sigma, u_{1}=v_{1} v, u_{2}=v v_{2}, v$ is a nonempty word, consider $w=v_{1} v v_{2}, g_{1}=f_{1} v_{2}, g_{2}=v_{1} f_{2}$.

In both cases, if there exit oriented paths $g_{1} \rightarrow \cdots \rightarrow h$ and $g_{2} \rightarrow \cdots \rightarrow h$ for an appropriate polynomial $h$ then we say that the composition of $u_{1} \rightarrow f_{1}$ and $u_{2} \rightarrow f_{2}$ relative to the word $w$ is confluent modulo $\Sigma$. Denote the polynomial $g_{1}-g_{2}$ by $\left(u_{1} \rightarrow f_{1}, u_{2} \rightarrow f_{2}\right)_{w}$.

Theorem 1 ([6, 7]). Suppose a set of rewriting rules $\Sigma$ in the free associative algebra $\mathbb{k}\langle B\rangle$ defines a rewriting system $\mathcal{G}(B, \Sigma)$. If every composition of rewriting rules from $\Sigma$ is confluent modulo $\Sigma$ then $\mathcal{G}(B, \Sigma)$ is a confluent rewriting system, i.e., $\Sigma$ is a $G S B$.

Let $\Sigma$ respect a monomial order $\leq$ on $B^{*}$. Then $\mathcal{G}(B, \Sigma)$ is a rewriting system and the confluence of a composition may be replaced with a more convenient condition.

Corollary 1 ([7]). If for every rewriting rules $u_{1} \rightarrow f_{1}, u_{2} \rightarrow f_{2}$ in $\Sigma$ having a composition relative to a word $w$ the polynomial $\left(u_{1} \rightarrow f_{1}, u_{2} \rightarrow f_{2}\right)_{w}$ may be presented as

$$
\sum_{i} \alpha_{i} w_{i}\left(u^{(i)}-f^{(i)}\right) w_{i}^{\prime}, \quad \alpha_{i} \in \mathbb{k}, w_{i}, w_{i}^{\prime} \in B^{*},
$$

where $u^{(i)} \rightarrow f^{(i)}$ in $\Sigma$ and $w_{i} u^{(i)} w_{i}^{\prime}<w$, then $\Sigma$ is a GSB.

In the actual computation, we will often apply the following trick to show the confluence of a fork $w \rightarrow g_{1}, w \rightarrow g_{2}$ : find some paths $g_{1} \rightarrow \cdots \rightarrow h_{1}$ and $g_{2} \rightarrow$ $\cdots \rightarrow h_{2}$ and then present $h_{1}-h_{2}$ in the form (4).

3.2. Rewriting system for bimodules over associative algebras. Let $A$ be an associative algebra (with a unit) and let $M$ be a bimodule over $A$. Suppose $A$ is generated by a subset $B \subset A$ as an algebra and $M$ is generated by a subset $Y$ as an $A$-module. Then $A$ is isomorphic to a quotient of the free associative algebra $\mathbb{k}\langle B\rangle$ modulo an ideal generated by a set of defining relations $R \subset \mathbb{k}\langle B\rangle$, i.e.,

$$
A \simeq \mathbb{k}\langle B \mid R\rangle
$$


Similarly, $M$ is a quotient of the free $A$-module $A \otimes \mathbb{k} Y \otimes A$ generated by $Y$ modulo a family of defining relations $S$. One may identify an element of $S$ with a noncommutative polynomial in the variables $B \cup Y$ which is linear in $Y$.

The split null extension $A \oplus M$ is an associative algebra isomorphic to the quotient of the free algebra generated by $B \cup Y$ modulo the ideal generated by the union of $R, S$, and

$$
y b_{1} \ldots b_{n} z, \quad y, z \in Y, b_{i} \in B, n \geq 0 .
$$

These relations reflect the properties of multiplication in $A \oplus M: M^{2}=0$.

Remark 1. To consider left modules, it is enough to add relations $y b, y \in Y, b \in B$ to reflect $M A=0$.

Suppose we may choose a monomial $u$ in each defining relation $u-f$ of $A \oplus M$ (up to a scalar multiple) in such a way that the family $\Sigma$ of all rewriting rules $u \rightarrow f$ defines a rewriting system $\mathcal{G}(B \cup Y, \Sigma)$. Note that the defining relations of $A \oplus M$ are homogeneous relative to $Y$. All monomials that are of degree $\geq 2$ in $Y$ belong to the same connected component as zero, so it is enough to consider only the relations of degree 0 and 1 in $Y$, these are exactly the defining relations of $A$ and of $M$, respectively. Therefore, the confluence test needs to be applied to the forks started at a word $w$ which either belongs to $B^{*}$ or contains only one letter from $Y$. Hence, the compositions emerging in this rewriting system are exactly those described in [17].

3.3. Free associative conformal algebras. Recall the construction of a free associative conformal algebra $\operatorname{Conf}(X, N)$ generated by a set $X$ relative to a given locality function $N: X \times X \rightarrow \mathbb{Z}_{+}$. From now on, denote by $H$ the polynomial algebra $\mathbb{k}[\partial]$.

By definition, $\operatorname{Conf}(X, N)$ is an associative conformal algebra generated by $X$ which is universal in the class of all associative conformal algebras $C$ generated by $X$ such that the mutual locality of elements from $X$ in $C$ is bounded by $N$. Namely, for every associative conformal algebra $C$ and for every map $\alpha: X \rightarrow C$ such that $N_{C}(\alpha(x), \alpha(y)) \leq N(x, y)$ for all $x, y \in X$ there exists unique homomorphism of conformal algebras $\varphi: \operatorname{Conf}(X, N) \rightarrow C$ such that $\varphi(x)=\alpha(x)$ for all $x \in X$.

Proposition 1 ([25]). The free associative conformal algebra $\operatorname{Conf}(X, N)$ is a free $H$-module with a basis

$$
\begin{gathered}
a_{1\left(n_{1}\right)}\left(a_{2\left(n_{2}\right)}\left(a_{3\left(n_{3}\right)} \cdots{ }_{\left(n_{k-1}\right)}\left(a_{k\left(n_{k}\right)} a_{k+1}\right) \ldots\right)\right), \\
a_{i} \in X, \quad 0 \leq n_{i} \leq N\left(a_{i}, a_{i+1}\right), k \in \mathbb{Z}_{+} .
\end{gathered}
$$

Remark 2. In a similar way, one may define the free associative commutative conformal algebra $\operatorname{Com} \operatorname{Conf}(X, N)$ generated by a set $X$ relative to a locality function $N$ [26]. However, there was no explicit description of a linear basis of $\operatorname{Com} \operatorname{Conf}(X, N)$ for $N>1$. We will obtain such a description for $N=2,3$ as a byproduct in Section 4 . 
The conformal algebra $\operatorname{Conf}(X, N)$ may be presented in a more convenient form as a (left) module over an appropriate associative algebra [20]. Given a set $X$, let $A(X)$ denote the associative algebra generated by the set

$$
B=\{\partial\} \cup\left\{L_{n}^{a}, R_{n}^{a} \mid a \in X, n \in \mathbb{Z}_{+}\right\}
$$

relative to the defining relations

$$
\begin{aligned}
& L_{n}^{a} \partial-\partial L_{n}^{a}-n L_{n-1}^{a}, \\
& R_{n}^{a} \partial-\partial R_{n}^{a}-n R_{n-1}^{a}, \\
& R_{n}^{a} L_{m}^{b}-L_{m}^{b} R_{n}^{a},
\end{aligned}
$$

where $a, b \in X, n, m \in \mathbb{Z}_{+}$.

The free associative conformal algebra $\operatorname{Conf}(X, N)$ is a left module over $A(X)$ if we define the action as follows:

$$
L_{n}^{a} u=a_{(n)} u, \quad R_{n}^{a} u=\left\{u_{(n)} a\right\}
$$

for $a \in X, n \in \mathbb{Z}_{+}, u \in \operatorname{Conf}(X, N)$. Therefore, $\operatorname{Conf}(X, N)$ considered as a left $A(X)$-module is a homomorphic image the free left $A(X)$-module $M(X)$ generated by the set $X$. It is not hard to find explicitly the kernel of that homomorphism $M(X) \rightarrow \operatorname{Conf}(X, N)$.

Fix a function $N: X \times X \rightarrow \mathbb{Z}_{+}$and consider the quotient $M(X, N)$ of $M(X)$ relative to the $A(X)$-submodule generated by the following elements:

$$
\begin{aligned}
& L_{n}^{a} b, \quad n \geq N(a, b), \\
& R_{m}^{a} b-\sum_{s=0}^{N(b, a)-m}(-1)^{m+s} \frac{1}{s !} \partial^{s} L_{m+s}^{b} a, \quad m \in \mathbb{Z}_{+},
\end{aligned}
$$

where $a, b \in X$. Obviously, there is a homomorphism $M(X, N) \rightarrow \operatorname{Conf}(X, N)$ of $A(X)$-modules extending $x \mapsto x$. This homomorphism is actually an isomorphism since (51) and (8) imply the following relations in $M(X, N)$ :

$$
L_{n}^{a} L_{m}^{b} u+\sum_{q \geq 1}(-1)^{q}\left(\begin{array}{l}
n \\
q
\end{array}\right) L_{n-q}^{a} L_{m+q}^{b} u=0,
$$

where $a, b \in X, n \geq N(a, b), m \in \mathbb{Z}_{+}, u \in M(X)$.

Consider the relations (15)-(10) as rewriting rules in such a way that the first monomial is always a principal one. The terminal words in $M(X)$ of the rewriting system obtained are

$$
\partial^{s} L_{n_{1}}^{a_{1}} L_{n_{2}}^{a_{2}} \ldots L_{n_{k}}^{a_{k}} a_{k+1}, \quad k \in \mathbb{Z}_{+}, a_{i} \in X, 0 \leq n_{i}<N\left(a_{i}, a_{i+1}\right), \quad s \in \mathbb{Z}_{+} .
$$

The images of these words in $\operatorname{Conf}(X, N)$ are linearly independent by Proposition 1 , hence we obtain the following

Corollary 2 ([20]). The free associative conformal algebra $\operatorname{Conf}(X, N)$ is isomorphic to $M(X, N)$ as an $A(X)$-module. 
It follows from the definition of the action of $A(X)$ on $\operatorname{Conf}(X, N)$ that every conformal ideal of $\operatorname{Conf}(X, N)$ is an $A(X)$-submodule and vice versa. Hence we may replace the study of conformal ideals with the study of "ordinary" submodules.

Example 1 ([8]). Let us determine the structure of an associative conformal algebra $C$ generated by the set $X=\{a\}$ relative to $N=N(a, a)=2$ with one defining relation $a_{(1)} a-\partial\left(a_{(0)} a\right)$.

The algebra $A(X)$ is generated by $L_{n}=L_{n}^{a}, R_{n}=R_{n}^{a}$, and $\partial$ satisfying (5). Namely, consider these relations as rewriting rules

$$
L_{n} \partial \rightarrow \partial L_{n}+n L_{n-1}, \quad R_{n} \partial \rightarrow \partial R_{n}+n R_{n-1}, \quad R_{n} L_{m} \rightarrow L_{m} R_{n} .
$$

Similarly, define the free conformal algebra $\operatorname{Conf}(X, N)$ as a module over $A(X)$ generated by a single element $a$ relative to the following rewriting rules (8)

$$
L_{n} a \rightarrow 0, R_{n} a \rightarrow 0, n \geq 2, R_{1} a \rightarrow-L_{1} a, \quad R_{0} a \rightarrow L_{0} a-\partial L_{1} a .
$$

The compositions (10) of these relations include

$$
L_{3} L_{1} \rightarrow 0, L_{3} L_{0} a \rightarrow 0, \quad L_{2} L_{1} \rightarrow 0, \quad L_{2} L_{0} \rightarrow 2 L_{1} L_{1} .
$$

The defining relation $a(1) a-\partial\left(a_{(0)} a\right)$ is naturally written as

$$
L_{1} a \rightarrow \partial L_{0} a .
$$

Consider the composition of $R_{2} L_{1} \rightarrow L_{1} R_{2}$ and (11) relative to $w=R_{2} L_{1} a$. On the one hand,

$$
R_{2} L_{1} a \rightarrow L_{1} R_{2} a \rightarrow 0
$$

on the other hand,

$$
R_{2} L_{1} a \rightarrow R_{2} \partial L_{0} a \rightarrow \partial R_{2} L_{0} a+2 R_{1} L_{0} a \rightarrow 2 L_{0} R_{1} a \rightarrow-2 L_{0} L_{1} a .
$$

Hence, we should add a new rewriting rule

$$
L_{0} L_{1} a \rightarrow 0 .
$$

The latter has a composition with (11) relative to $w=L_{0} L_{1} a$ :

$$
L_{0} L_{1} a \rightarrow L_{0} \partial L_{0} a \rightarrow \partial L_{0} L_{0} a
$$

Hence, we should add

$$
\partial L_{0} L_{0} a \rightarrow 0 \text {. }
$$

Next, consider the composition of $R_{1} L_{1} \rightarrow L_{1} R_{1}$ and (11) relative to $w=R_{1} L_{1} a$. In a similar way, we obtain that $-L_{1} L_{1} a$ and $L_{0} L_{0} a$ are connected by a (nonoriented) path, so add

$$
L_{1} L_{1} a \rightarrow-L_{0} L_{0} a
$$

(the choice of the principal part is voluntary since we have not fixed an order on the words). 
Let us calculate the composition of $R_{0} L_{1} a \rightarrow L_{1} R_{0} a$ and (11) relative to $w=$ $R_{0} L_{1} a$ in more details:

$$
\begin{aligned}
R_{0} L_{1} a \rightarrow L_{1} R_{0} a & \rightarrow L_{1} L_{0} a-L_{1} \partial L_{1} a \rightarrow L_{1} L_{0}-\partial L_{1} L_{1} a-L_{0} L_{1} a \\
& \rightarrow L_{1} L_{0} a+\partial L_{0} L_{0} a \rightarrow L_{1} L_{0} a \\
R_{0} L_{1} a & \rightarrow R_{0} \partial L_{0} a \rightarrow \partial L_{0} R_{0} a \rightarrow \partial L_{0} L_{0} a-\partial L_{0} \partial L_{1} a \\
& \rightarrow \partial L_{0} L_{0} a-\partial^{2} L_{0} L_{1} a \rightarrow \partial L_{0} L_{0} a \rightarrow 0 .
\end{aligned}
$$

Hence, we should add

$$
L_{1} L_{0} a \rightarrow 0 .
$$

There exist compositions between (10) and (11). For example, the composition of $L_{2} L_{1} a \rightarrow 0$ and (11) is trivial:

$$
L_{2} L_{1} a \rightarrow L_{2} \partial L_{0} a \rightarrow \partial L_{2} L_{0} a+2 L_{1} L_{0} a \rightarrow 2 \partial L_{1} L_{1} a \rightarrow-2 \partial L_{0} L_{0} a \rightarrow 0 .
$$

However, the composition of (11) and $L_{3} L_{1} a \rightarrow 0$ is not trivial:

$$
L_{3} L_{1} a \rightarrow L_{3} \partial L_{0} a \rightarrow \partial L_{3} L_{0} a+3 L_{2} L_{0} a \rightarrow 6 L_{1} L_{1} a .
$$

Hence, we should add

$$
L_{1} L_{1} a \rightarrow 0
$$

and (13) implies

$$
L_{0} L_{0} a \rightarrow 0 \text {. }
$$

The relations (12), (14), (15), (16) along with (8) and (10) form a GröbnerShirshov basis of $C$ as of $A(X)$-module: all other compositions are trivial by homogeneity reasons. As a result, the basis of $C$ as of a module over $H=\mathbb{k}[\partial]$ consists of two elements: $a$ and $a_{(0)} a$, all words of degree $\geq 3$ are zero.

3.4. Universal associative conformal envelopes of Lie conformal algebras. Suppose $L$ is a Lie conformal algebra generated by a set $X$. Thus $L$ is a quotient of an appropriate free Lie conformal algebra by the ideal generated by a set $\Sigma$ of defining relations stated in terms of Lie conformal operations $\left[x_{(n)} y\right]$. The structure of free Lie conformal algebras was described in [25].

For a given function $N: X \times X \rightarrow \mathbb{Z}_{+}$, the universal enveloping associative conformal algebra $U(L ; X, N)$ of $L$ relative to the locality level $N$ on $X$ is defined as the quotient of $\operatorname{Conf}(X, N)$ relative to the same defining relations $\Sigma$ rewritten by the rules

$$
\left[x_{(n)} y\right]=\left(x_{(n)} y\right)-\sum_{s \geq 0} \frac{(-1)^{n+s}}{s !} \partial^{s}\left(y_{(n+s)} x\right),
$$

where the upper limit of the summation is determined by the Dong Lemma.

The main purpose of this paper is to study universal enveloping associative conformal algebras for Kac-Moody conformal algebras. The latter are central extensions of current Lie conformal algebras. For this particular class of problems, the Gröbner-Shirshov bases method described above may be slightly modified. The main advantage of the modification is that the relations (10) become not necessary. 
Suppose $L$ is a Lie conformal algebra with an $H$-torsion $L_{0}$ such that the torsionfree $L_{1}=L / L_{0}$ is a free $H$-module (for example, every finite Lie conformal algebra has that property). Assume $X=X_{1} \cup X_{0}$, where $X_{1}$ is an $H$-basis of $L_{1}$ and $X_{0}$ is a $\mathbb{k}$-basis of $X_{0}$. Then the structure of $L$ is completely determined by relations

$$
f_{e}(\partial) e=0, \quad e \in X_{0}
$$

and

$$
\left[x_{(n)} y\right]=\sum_{z \in X_{1}} f_{x, y}^{n, z}(\partial) z+\sum_{e \in X_{0}} g_{x, y}^{n, e}(\partial) e, \quad x, y \in X_{1}
$$

for appropriate $f_{e}, f_{x, y}^{n, z}, g_{x, y}^{n, e} \in \mathbb{k}[\partial]$. These relations describe the structure of $L_{0}$ as of a torsion $H$-module, the multiplication table in the Lie conformal algebra $L_{1}$, and the structure of the extension

$$
0 \rightarrow L_{0} \rightarrow L \rightarrow L_{1} \rightarrow 0
$$

Then, for a given function $N: X_{1} \times X_{1} \rightarrow \mathbb{Z}_{+}$, the conformal algebra $U(L ; X, N)$ may be considered as an ordinary left module over the associative algebra $A(X ; L)$ generated by

$$
\left\{\partial, L_{n}^{x}, R_{n}^{x} \mid n \in \mathbb{Z}_{+}, x \in X_{1}\right\}
$$

relative to defining relations (5) (for $a \in X_{1}$ ) along with the following ones:

$$
L_{n}^{x} L_{m}^{y}-L_{m}^{y} L_{n}^{x}-\sum_{s \geq 0} \sum_{z \in X_{1}} L_{n+m-s}^{f_{x, y}^{n, z}(\partial) z}
$$

where $L_{n}^{\partial z}$ is naturally understood as $-n L_{n-1}^{z}$. The relations (17) reflect the property (3) of associative conformal algebras. So $U(L ; X, N)$ is a left module over $A(X ; L)$ generated by the entire set $X$ relative to the relations (8) (for $a, b \in X_{1}$ ) together with

$$
\begin{gathered}
f_{e}(\partial) e, \quad e \in X_{0}, \\
L_{n}^{a} e, R_{n}^{a} e \quad a \in X_{1}, e \in X_{0}, n \in \mathbb{Z}_{+}, \\
R_{n}^{a} b-L_{n}^{a} b+\left[a_{(n)} b\right], \quad a, b \in X_{1} .
\end{gathered}
$$

Since the defining relations of $A(X ; L)$ already form a Gröbner-Shirshov basis, in order to determine the structure of $U(L ; X, N)$ one needs to find a confluent system of rewriting rules in this $A(X ; L)$-module. In the next section, we solve this problem for a Kac-Moody conformal algebra.

\section{The Poincaré-Birkhoff-Witt Theorem for KaC-Moody CONFORMAL ALGEBRAS AT $N=3$}

Let $\mathfrak{g}$ be a Lie algebra and let $\langle\cdot \mid \cdot\rangle$ be a bilinear symmetric invariant form on $\mathfrak{g}$ (e.g., the Killing form). Then $K(\mathfrak{g})=(\mathbb{k}[\partial] \otimes \mathfrak{g}) \oplus \mathbb{k} e$, where $\partial e=0$, equipped with

$$
\left[a_{(\lambda)} b\right]=[a, b]+\lambda\langle a \mid b\rangle, \quad\left[a_{(\lambda)} e\right]=\left[e_{(\lambda)} e\right]=0
$$

for every $a, b \in \mathfrak{g}$ is a Lie conformal algebra with 1-dimensional torsion $\mathbb{k} e$ and the torsion-free image isomorphic to Cur $\mathfrak{g}$. 
Let us fix a linear basis $X_{1}$ of $\mathfrak{g}$. Then $X=X_{1} \cup\{e\}$ is a generating set of $K(\mathfrak{g})$. The purpose of this section is to calculate the Gröbner-Shirshov basis for $U=U(K(\mathfrak{g}) ; X, N)$ for $N=3$ and prove the Poincaré-Birkhoff-Witt Theorem for this universal enveloping associative conformal algebra.

According to the scheme described in the previous section, $U$ is a module over the associative algebra $A=A(X ; K(\mathfrak{g}))$ generated by the set $B=\left\{\partial, L_{n}^{a}, R_{n}^{a} \mid a \in\right.$ $\left.X_{1}, n \in \mathbb{Z}_{+}\right\}$modulo the relations

$$
\begin{gathered}
L_{n}^{a} \partial-\partial L_{n}^{a}-n L_{n-1}^{a}, \quad R_{n}^{a} \partial-\partial R_{n}^{a}-n R_{n-1}^{a}, \quad R_{n}^{a} L_{m}^{b}-L_{m}^{b} R_{n}^{a} \\
L_{n}^{a} L_{m}^{b}-L_{m}^{b} L_{n}^{a}-L_{n+m}^{[a, b]} .
\end{gathered}
$$

The set of generators of $U$ as of an $A$-module is $X=X_{1} \cup\{e\}$, and the defining relations of this module are

$$
\begin{gathered}
L_{n}^{a} b, R_{n}^{a} b \quad n \geq N=3, \\
L_{n}^{a} e, R_{n}^{a} e, \quad n \geq 0 \\
R_{2}^{a} b-L_{2}^{b} a, \quad R_{1}^{a} b+L_{1}^{b} a-\partial L_{2}^{b} a, \\
R_{0}^{a} b-L_{0}^{b} a+\partial L_{1}^{b} a-\frac{1}{2} \partial^{2} L_{2}^{b} a, \\
R_{0}^{a} b-L_{0}^{a} b+[a, b], \quad R_{1}^{a} b-L_{1}^{a} b+\langle a \mid b\rangle e, \quad R_{2}^{a} b-L_{2}^{a} b,
\end{gathered}
$$

for all $a, b \in X_{1}$.

In order to translate these defining relations into rewriting rules we need to choose a principal monomial in each relation. The choice of principal parts affects on the resulting system of rewriting rules obtained in a process of adding compositions similar to Example 11.

We will always choose a principal term in a rewriting rule as a leading monomial relative to an appropriate order $\leq$ on the monomials in the free $\mathbb{k}\langle B\rangle$-module generated by $X$. Namely, suppose the set $X_{1}$ is linearly well ordered and $e<X_{1}$. Induce an order on $B$ by the rule

$$
L_{0}^{a}<L_{1}^{a}<\partial<L_{2}^{a}<\cdots<R_{0}^{a}<R_{1}^{a}<\ldots
$$

assuming $L_{n}^{a}<R_{m}^{b}$ and $L_{n}^{a}<L_{n}^{b}$ iff $a<b$, for $a, b \in X_{1}$ (this ordering turns to be the most convenient for our purpose). Extend the order on the set of monomials in $B^{*}$ by the deg-lex principle, i.e., first compare the lengths and then lexicographically.

For two monomials $u x$ and $v y$ in $\mathbb{k}\langle B\rangle X, u, v \in B^{*}, x, y \in X$, set $u x<v y$ iff $(u, x)$ is lexicographically less than $(v, y)$.

Then, eliminating the monomials $R_{n}^{a} b(n=0,1,2)$ in (25) and (26) by means of (27), we obtain the following set of rewriting rules defining $U$ (along with the 
confluent set of rewriting rules for $A)$ :

$$
\begin{gathered}
\partial L_{0}^{a} \rightarrow L_{0}^{a} \partial, \quad \partial L_{1}^{a} \rightarrow L_{1}^{a} \partial-L_{0}^{a}, \\
L_{n}^{a} \partial \rightarrow \partial L_{0}^{a}+n L_{n-1}^{a}, \quad n \geq 2, \\
R_{n}^{a} \partial \rightarrow \partial R_{n}^{a}+n R_{n-1}^{a}, \quad n \geq 0, \\
R_{m}^{a} L_{n}^{b} \rightarrow L_{n}^{b} R_{m}^{a}, \quad n, m \geq 0, \\
L_{n}^{a} L_{m}^{b} \rightarrow L_{m}^{b} L_{n}^{a}+L_{n+m}^{[a, b]}, \quad(n, a)>_{l e x}(m, b) ; \\
L_{n}^{a} b \rightarrow 0, \quad R_{n}^{a} \rightarrow 0, \quad n \geq 3, \\
R_{2}^{a} b \rightarrow L_{2}^{a} b, \quad R_{1}^{a} b \rightarrow L_{1}^{a} b-\langle a \mid b\rangle e, \\
R_{0}^{a} b \rightarrow L_{0}^{a} b-[a, b] ; \\
L_{2}^{a} b \rightarrow L_{2}^{b} a, \quad a>b, \\
\partial L_{2}^{a} b \rightarrow L_{1}^{a} b+L_{1}^{b} a-\langle a \mid b\rangle e, \\
L_{1}^{a} \partial b \rightarrow L_{1}^{b} \partial a+3 L_{0}^{a} b-3 L_{0}^{b} a-2[a, b], \quad a>b .
\end{gathered}
$$

Theorem 2. The set of rewriting rules (28)-(30) along with

$$
\begin{gathered}
L_{1}^{a} \partial^{s} b \rightarrow L_{1}^{b} \partial^{s} a-(s+2) L_{0}^{b} \partial^{s-1} a+(s+2) L_{0}^{a} \partial^{s-1} b-2 \partial^{s-1}[a, b], \\
s \geq 2, a>b, \\
L_{2}^{a} L_{2}^{b} c \rightarrow 0, \quad a, b, c \in X_{1} \\
L_{1}^{a} L_{2}^{b} c \rightarrow L_{1}^{b} L_{2}^{c} a, \quad b \leq c<a, \\
L_{1}^{a} L_{2}^{b} c \rightarrow L_{1}^{b} L_{2}^{a} c, \quad b<a \leq c, \\
L_{1}^{a} L_{1}^{b} c \rightarrow L_{1}^{a} L_{1}^{c} b+L_{0}^{b} L_{2}^{a} c-L_{0}^{c} L_{2}^{a} b+L_{2}^{a}[c, b]+L_{2}^{b}[c, a]+L_{2}^{c}[a, b], \quad a \leq c<b, \\
L_{1}^{a} L_{1}^{b} c \rightarrow L_{1}^{c} L_{1}^{a} b+L_{0}^{b} L_{2}^{c} a-L_{0}^{c} L_{2}^{a} b+L_{2}^{c}[a, b]+L_{2}^{a}[c, b], \quad c<a \leq b, \\
L_{0}^{a} L_{1}^{b} c \rightarrow L_{0}^{a} L_{1}^{c} b+L_{0}^{b} L_{1}^{a} c+L_{0}^{c} L_{1}^{b} a-L_{0}^{b} L_{1}^{c} a-L_{0}^{c} L_{1}^{a} b+L_{1}^{[c, a]} b+\quad \\
+L_{1}^{[a, b]} c+L_{1}^{[b, c]} a-L_{1}^{c}[a, b]-L_{1}^{a}[b, c]-L_{1}^{b}[c, a]+\langle a \mid[b, c]\rangle e, \quad c<b<a,
\end{gathered}
$$

is a Gröbner-Shirshov basis of the universal associative envelope $U=U(K(\mathfrak{g}) ; X, 3)$.

Proof. First, we will show how to derive the rules (31)-(37) as compositions of the initial relations. Next, we will check the triviality of compositions obtained in further iterations.

Since the calculations are routine, we will state them in details for several particular cases, other cases are essentially the same and may be processed in a similar way.

For the purpose of clarity, we will use a brief notation to point a rule applied for rewriting (e.g., $(R L)$ stands for $R_{m}^{a} L_{n}^{b} \rightarrow L_{n}^{b} R_{m}^{a}, n, m \geq 0,\left(\partial L_{2}\right)$ for $\partial L_{2}^{a} b \rightarrow$ $L_{1}^{a} b+L_{1}^{b} a-\langle a \mid b\rangle e$, etc $)$. 
The rule (31) for $s=2$ appears from the intersection of $\left(\partial L_{1}\right)$ and $\left(L_{1} \partial\right)$. Then, by induction, the intersection with $\left(\partial L_{1}\right)$ produces (31) for $s>2$ :

$$
\begin{gathered}
\partial L_{1}^{a} \partial^{s} b \rightarrow \partial\left(L_{1}^{a} \partial^{s} b-(s+2) L_{0}^{b} \partial^{s-1} a+(s+2) L_{0}^{a} \partial^{s-1} b-2 \partial^{s-1}[a, b]\right) \\
\rightarrow L_{1}^{a} \partial^{s+1} b-(s+3) L_{0}^{b} \partial^{s} a+(s+2) L_{0}^{a} \partial^{s} b-2 \partial^{s}[a, b] ; \\
\partial L_{1}^{a} \partial^{s} b \rightarrow L_{1}^{a} \partial^{s+1} b-L_{0}^{a} \partial^{s} b .
\end{gathered}
$$

The rule (32) fairly simply derives from $\left(\partial L_{2}\right)$ in (30) and $\left(L_{n} \partial\right)$ for $n=2$.

The next example of an intersection of $\left(L_{1} \partial\right)$ and $(R L)$ produces the rest of the required rules. On the one hand, we have

$$
R_{n}^{a} L_{1}^{b} \partial c \stackrel{(R L)}{\rightarrow} L_{1}^{b} R_{n}^{a} \partial c \stackrel{(R \partial)}{\rightarrow} L_{1}^{b} \partial R_{n}^{a} c+n L_{1}^{b} R_{n-1}^{a} c
$$

on the other hand,

$$
\begin{aligned}
& R_{n}^{a} L_{1}^{b} \partial c \stackrel{\left(L_{1} \partial\right)}{\rightarrow} R_{n}^{a} L_{1}^{c} \partial b-3 R_{n}^{a} L_{0}^{c} b+3 R_{n}^{a} L_{0}^{b} c-2 R_{n}^{a}[b, c] \stackrel{(R L)}{\rightarrow} L_{1}^{c} R_{n}^{a} \partial b-3 L_{0}^{c} R_{n}^{a} b \\
& +3 L_{0}^{b} R_{n}^{a} c-2 R_{n}^{a}[b, c] \stackrel{(R \partial)}{\rightarrow} L_{1}^{c} \partial R_{n}^{a} b+n L_{1}^{c} R_{n-1}^{a} b-3 L_{0}^{c} R_{n}^{a} b+3 L_{0}^{b} R_{n}^{a} c-2 R_{n}^{a}[b, c] .
\end{aligned}
$$

In order to apply $\left(L_{1} \partial\right)$ we have to assume $b>c$. However, the composition obtained by subtracting the right-hand sides of the two expressions above is

$$
L_{1}^{b} \partial R_{n}^{a} c+n L_{1}^{b} R_{n-1}^{a} c-L_{1}^{c} \partial R_{n}^{a} b-n L_{1}^{c} R_{n-1}^{a} b+3 L_{0}^{c} R_{n}^{a} b-3 L_{0}^{b} R_{n}^{a} c+2 R_{n}^{a}[b, c],
$$

it is (skew-)symmetric relative to the permutation of $b$ and $c$. Hence, we may assume the relation (38) holds on $U$ for every $a, b, c \in X_{1}$.

For $n \geq 4$ the composition is trivial due to the locality. For $n=3$, apply the rules $\left(R_{3}\right)$ and $\left(R_{2}\right)$ to get the following:

$$
L_{1}^{b} L_{2}^{a} c-L_{1}^{c} L_{2}^{a} b, \quad a, b, c \in X_{1} .
$$

For a fixed order on $a, b, c \in X_{1}$, use $\left(L_{2}\right)$ if necessary to obtain (33) or (34).

Consider (38) for $n=2$. For convenience of the exposition, let us split the polynomial into two summands and process the summands separately:

$$
\begin{gathered}
\left(L_{1}^{b} \partial R_{2}^{a} c+2 L_{1}^{b} R_{1}^{a} c\right)-\left(L_{1}^{c} \partial R_{2}^{a} b+2 L_{1}^{c} R_{1}^{a} b-3 L_{0}^{c} R_{2}^{a} b+3 L_{0}^{b} R_{2}^{a} c-2 R_{2}^{a}[b, c]\right), \quad \text { (40) } \\
L_{1}^{b} \partial R_{2}^{a} c+2 L_{1}^{b} R_{1}^{a} c \stackrel{\left(R_{1}\right),\left(R_{2}\right)}{\rightarrow} L_{1}^{b} \partial L_{2}^{a} c+2 L_{1}^{b} L_{1}^{a} c-2\langle a \mid c\rangle L_{1}^{b} e \\
\quad \stackrel{\left(\partial L_{2}\right),(L e)}{\rightarrow} L_{1}^{b} L_{1}^{a} c+L_{1}^{b} L_{1}^{c} a-\langle c \mid a\rangle L_{1}^{b} e+2 L_{1}^{b} L_{1}^{a} c \stackrel{(L L),(L e)}{\rightarrow} 3 L_{1}^{b} L_{1}^{a} c+L_{1}^{c} L_{1}^{b} a+L_{2}^{[b, c]} a, \\
L_{1}^{c} \partial R_{2}^{a} b+2 L_{1}^{c} R_{1}^{a} b-3 L_{0}^{c} R_{2}^{a} b+3 L_{0}^{b} R_{2}^{a} c-2 R_{2}^{a}[b, c] \stackrel{\left(R_{1}\right),\left(R_{2}\right)}{\rightarrow} L_{1}^{c} \partial L_{2}^{a} b+2 L_{1}^{c} L_{1}^{a} b \\
-2\langle a \mid b\rangle L_{1}^{c} e-3 L_{0}^{c} L_{2}^{a} b+3 L_{0}^{b} L_{2}^{a} c-2 L_{2}^{a}[b, c] \stackrel{\left(\partial L_{2}\right)_{(}(L e)}{\rightarrow} L_{1}^{c} L_{1}^{a} b+L_{1}^{c} L_{1}^{b} a-\langle b \mid a\rangle L_{1}^{c} e+2 L_{1}^{c} L_{1}^{a} b \\
-3 L_{0}^{c} L_{2}^{a} b+3 L_{0}^{b} L_{2}^{a} c-2 L_{2}^{a}[b, c] \stackrel{(L e)}{\rightarrow} 3 L_{1}^{c} L_{1}^{a} b+L_{1}^{c} L_{1}^{b} a-3 L_{0}^{c} L_{2}^{a} b+3 L_{0}^{b} L_{2}^{a} c-2 L_{2}^{a}[b, c] .
\end{gathered}
$$


Therefore, (40) modulo $\left(L_{2}\right)$ (i.e., $L_{2}^{[b, c]} a-L_{2}^{a}[b, c]$ ) implies the following relation:

$$
-L_{1}^{b} L_{1}^{a} c+L_{1}^{c} L_{1}^{a} b-L_{0}^{c} L_{2}^{a} b+L_{0}^{b} L_{2}^{a} c+L_{2}^{a}[c, b]
$$

for all $a, b, c \in X_{1}$.

Now we can switch $a$ and $b$ in (41) and subtract the relation obtained from (41). In this way, we actually apply the rule $(L L)$ without fixing an order on $a, b \in X_{1}$. As a result, we obtain

$$
\begin{aligned}
-L_{1}^{b} L_{1}^{a} c+L_{1}^{c} L_{1}^{a} b-L_{0}^{c} L_{2}^{a} b+L_{0}^{b} L_{2}^{a} c+L_{2}^{a}[c, b] & \\
& +L_{1}^{a} L_{1}^{b} c-L_{1}^{c} L_{1}^{b} a+L_{0}^{c} L_{2}^{b} a-L_{0}^{a} L_{2}^{b} c-L_{2}^{b}[c, a]
\end{aligned}
$$

Let us apply $(L L)$ and $\left(L_{2}\right)$ to write the last relation in a more convenient form:

$$
L_{1}^{c} L_{1}^{a} b-L_{1}^{c} L_{1}^{b} a+L_{0}^{b} L_{2}^{a} c-L_{0}^{a} L_{2}^{b} c+L_{2}^{c}[a, b]+L_{2}^{a}[c, b]+L_{2}^{b}[a, c] .
$$

Given a fixed order on $a, b, c \in X_{1}$, use $(L L)$ and $\left(L_{2}\right)$ if necessary to obtain (35) or (36).

For $n=1$, proceed with (38) in a similar way:

$$
\left(L_{1}^{b} \partial R_{1}^{a} c+L_{1}^{b} R_{0}^{a} c\right)-\left(L_{1}^{c} \partial R_{1}^{a} b+L_{1}^{c} R_{0}^{a} b-3 L_{0}^{c} R_{1}^{a} b+3 L_{0}^{b} R_{1}^{a} c-2 R_{1}^{a}[b, c]\right) .
$$

On the one summand, we have

$$
\begin{aligned}
& L_{1}^{b} \partial R_{1}^{a} c+L_{1}^{b} R_{0}^{a} c \stackrel{\left(R_{0}\right),\left(R_{1}\right)}{\rightarrow} L_{1}^{b} \partial L_{1}^{a} c+L_{1}^{b} L_{0}^{a} c-L_{1}^{b}[a, c] \stackrel{\left(\partial L_{1}\right)}{\rightarrow} L_{1}^{b} L_{1}^{a} \partial c-L_{1}^{b} L_{0}^{a} c \\
& +L_{1}^{b} L_{0}^{a} c-L_{1}^{b}[a, c] \stackrel{\left(L_{1} \partial\right)}{\rightarrow} L_{1}^{b} L_{1}^{c} \partial a-3 L_{1}^{b} L_{0}^{c} a+3 L_{1}^{b} L_{0}^{a} c-2 L_{1}^{b}[a, c]-L_{1}^{b}[a, c] \\
& \quad \stackrel{(L L)}{\rightarrow} L_{1}^{c} L_{1}^{b} \partial a+L_{2}^{[b, c]} \partial a-3 L_{0}^{c} L_{1}^{b} a-3 L_{1}^{[b, c]} a+3 L_{0}^{a} L_{1}^{b} c+3 L_{1}^{[b, a]} c-3 L_{1}^{b}[a, c],
\end{aligned}
$$

whereas on the other

$$
\begin{gathered}
L_{1}^{c} \partial R_{1}^{a} b+L_{1}^{c} R_{0}^{a} b-3 L_{0}^{c} R_{1}^{a} b+3 L_{0}^{b} R_{1}^{a} c-2 R_{1}^{a}[b, c] \stackrel{\left(R_{0}\right),\left(R_{1}\right)}{\rightarrow} L_{1}^{c} \partial L_{1}^{a} b+L_{1}^{c} L_{0}^{a} b-L_{1}^{c}[a, b] \\
-3 L_{0}^{c} L_{1}^{a} b+3 L_{0}^{b} L_{1}^{a} c-2 L_{1}^{a}[b, c]+2\langle a \mid[b, c]\rangle e \stackrel{\left(\partial L_{1}\right)}{\rightarrow} L_{1}^{c} L_{1}^{a} \partial b-L_{1}^{c} L_{0}^{a} b+L_{1}^{c} L_{0}^{a} b-L_{1}^{c}[a, b] \\
-3 L_{0}^{c} L_{1}^{a} b+3 L_{0}^{b} L_{1}^{a} c-2 L_{1}^{a}[b, c]+2\langle a \mid[b, c]\rangle e \stackrel{\left(L_{1} \partial\right)}{\rightarrow} L_{1}^{c} L_{1}^{b} \partial a-3 L_{1}^{c} L_{0}^{b} a+3 L_{1}^{c} L_{0}^{a} b-2 L_{1}^{c}[a, b] \\
-L_{1}^{c}[a, b]-3 L_{0}^{c} L_{1}^{a} b+3 L_{0}^{b} L_{1}^{a} c-2 L_{1}^{a}[b, c]+2\langle a \mid[b, c]\rangle e \stackrel{(L L)}{\rightarrow} L_{1}^{c} L_{1}^{b} \partial a-3 L_{0}^{b} L_{1}^{c} a-3 L_{1}^{[c, b]} a \\
+3 L_{0}^{a} L_{1}^{c} b+3 L_{1}^{[c, a]} b-3 L_{1}^{c}[a, b]-3 L_{0}^{c} L_{1}^{a} b+3 L_{0}^{b} L_{1}^{a} c-2 L_{1}^{a}[b, c]+2\langle a \mid[b, c]\rangle e .
\end{gathered}
$$

Once the expressions subtracted, the terms $L_{1}^{c} L_{1}^{b} \partial a$ cancel among other similar terms, and the result may be rewritten via $\left(L_{2} \partial\right)$ and $\left(\partial L_{2}\right)$. The resulting expression is symmetric in $a, b, c \in X_{1}$, so as we fix the order $c<b<a$ the principal part of the relation obtained is $L_{0}^{a} L_{1}^{b} c$, and the rule (37) follows.

For $n=0$, the relation (38) turns into

$$
L_{1}^{b} \partial R_{0}^{a} c-\left(L_{1}^{c} \partial R_{0}^{a} b-3 L_{0}^{c} R_{0}^{a} b+3 L_{0}^{b} R_{0}^{a} c-2 R_{0}^{a}[b, c]\right) .
$$


Without loss of generality, assume $b>c$. Then the two summands in the above relation may be rewritten as follows:

$$
\begin{gathered}
L_{1}^{b} \partial R_{0}^{a} c \stackrel{\left(R_{0}\right)}{\rightarrow} L_{1}^{b} \partial L_{0}^{a} c-L_{1}^{b} \partial[a, c] \stackrel{\left(\partial L_{0}\right),(L L)}{\rightarrow} L_{0}^{a} L_{1}^{b} \partial c+L_{1}^{[b, a]} \partial c-L_{1}^{b} \partial[a, c] \\
\stackrel{\left(L_{1} \partial\right)}{\rightarrow} L_{0}^{a} L_{1}^{c} \partial b-3 L_{0}^{a} L_{0}^{c} b+3 L_{0}^{a} L_{0}^{b} c-2 L_{0}^{a}[b, c]+L_{1}^{c} \partial[b, a]-3 L_{0}^{c}[b, a]+3 L_{0}^{[b, a]} c- \\
2[[b, a], c]-L_{1}^{[a, c]} \partial b+3 L_{0}^{[a, c]} b-3 L_{0}^{b}[a, c]+2[b,[a, c]], \\
L_{1}^{c} \partial R_{0}^{a} b-3 L_{0}^{c} R_{0}^{a} b+3 L_{0}^{b} R_{0}^{a} c-2 R_{0}^{a}[b, c] \stackrel{\left(R_{0}\right)}{\rightarrow} L_{1}^{c} \partial L_{0}^{a} b-L_{1}^{c} \partial[a, b]-3 L_{0}^{c} L_{0}^{a} b+ \\
3 L_{0}^{c}[a, b]+3 L_{0}^{b} L_{0}^{a} c-3 L_{0}^{b}[a, c]-2 L_{0}^{a}[b, c]+2[a,[b, c]] \\
\stackrel{\left(\partial L_{0}\right),(L L)}{\rightarrow} L_{0}^{a} L_{1}^{c} \partial b+L_{1}^{[c, a]} \partial b-L_{1}^{c} \partial[a, b]-3 L_{0}^{a} L_{0}^{c} b-3 L_{0}^{[c, a]} b+3 L_{0}^{c}[a, b] \\
+3 L_{0}^{a} L_{0}^{b} c+3 L_{0}^{[b, a]} c-3 L_{0}^{b}[a, c]-2 L_{0}^{a}[b, c]+2[a,[b, c]] .
\end{gathered}
$$

Hence, the composition (38) for $n=0$ is trivial due to the skew-symmetry and the Jacobi identity on $[\cdot, \cdot]$.

In order to finish the proof one needs to check that the family of rewriting rules obtained is complete, i.e., forms a Gröbner-Shirshov basis. Let us consider several intersections as examples, other possible intersections may be processed in a similar way.

As a first example, consider the intersection of (33) and $\left(\partial L_{1}\right)$ :

$$
\begin{aligned}
\partial L_{1}^{a} L_{2}^{b} c \rightarrow \partial L_{1}^{b} L_{2}^{c} a \rightarrow L_{1}^{b} \partial L_{2}^{c} a-L_{0}^{b} L_{2}^{c} a \rightarrow L_{1}^{b} L_{1}^{c} a+L_{1}^{b} L_{1}^{a} c-L_{0}^{b} L_{2}^{c} a, \\
\partial L_{1}^{a} L_{2}^{b} c \rightarrow L_{1}^{a} L_{2}^{b} c-L_{0}^{a} L_{2}^{b} c \rightarrow L_{1}^{a} L_{1}^{b} c+L_{1}^{a} L_{1}^{c} b-L_{0}^{a} L_{2}^{b} c \\
\rightarrow L_{1}^{b} L_{1}^{a} c+L_{1}^{c} L_{1}^{a} b-L_{0}^{a} L_{2}^{b} c+L_{2}^{[a, b]} c+L_{2}^{[a, c]} b .
\end{aligned}
$$

Subtract the relations obtained to get a composition

$$
L_{1}^{c} L_{1}^{a} b-L_{1}^{b} L_{1}^{c} a+L_{0}^{b} L_{2}^{c} a-L_{0}^{a} L_{2}^{b} c+L_{2}^{[a, b]} c+L_{2}^{[a, c]} b, \quad b \leq c<a .
$$

If $b<c$ then apply (36) to rewrite the composition (43) into

$$
L_{2}^{b}[c, a]+L_{2}^{c}[b, a]+L_{2}^{[a, b]} c+L_{2}^{[a, c]} b .
$$

The latter reduces to zero by $\left(L_{2}\right)$. If $b=c$ in then apply (35) and $\left(L_{2}\right)$ to reduce (43) to zero.

As a more complicated example, consider the intersection of $(R L)$ with (35). On the one hand,

$$
R_{n}^{d} L_{1}^{a} L_{1}^{b} c \stackrel{(R L)}{\rightarrow} L_{1}^{a} L_{1}^{b} R_{n}^{d} c
$$


on the other hand,

$$
\begin{aligned}
& R_{n}^{d} L_{1}^{a} L_{1}^{b} c \stackrel{(35)}{\rightarrow} R_{n}^{d} L_{1}^{a} L_{1}^{c} b+R_{n}^{d} L_{0}^{b} L_{2}^{a} c-R_{n}^{d} L_{0}^{c} L_{2}^{a} b+R_{n}^{d} L_{2}^{a}[b, c]+R_{n}^{d} L_{2}^{b}[c, a]+R_{n}^{d} L_{2}^{c}[a, b] \\
& \stackrel{(R L)}{\rightarrow} L_{1}^{a} L_{1}^{c} R_{n}^{d} b+L_{0}^{b} L_{2}^{a} R_{n}^{d} c-L_{0}^{c} L_{2}^{a} R_{n}^{d} b+L_{2}^{a} R_{n}^{d}[b, c]+L_{2}^{b} R_{n}^{d}[c, a]+L_{2}^{c} R_{n}^{d}[a, b] .
\end{aligned}
$$

Here $a \leq c<b$, as in (35).

The composition obtained should be considered for different $n$ 's. If $n \geq 3$ then all terms reduce to zero by the locality. For $n=2$ we have

$$
\begin{array}{r}
-L_{1}^{a} L_{1}^{b} R_{2}^{d} c+L_{1}^{a} L_{1}^{c} R_{2}^{d} b+L_{0}^{b} L_{2}^{a} R_{2}^{d} c-L_{0}^{c} L_{2}^{a} R_{2}^{d} b+L_{2}^{a} R_{2}^{d}[c, b]+L_{2}^{b} R_{2}^{d}[c, a]+L_{2}^{c} R_{2}^{d}[a, b] \\
\stackrel{\left(R_{2}\right)}{\rightarrow}-L_{1}^{a} L_{1}^{b} L_{2}^{d} c+L_{1}^{a} L_{1}^{c} L_{2}^{d} b+L_{0}^{b} L_{2}^{a} L_{2}^{d} c-L_{0}^{c} L_{2}^{a} L_{2}^{d} b+L_{2}^{a} L_{2}^{d}[c, b]+L_{2}^{b} L_{2}^{d}[c, a]+L_{2}^{c} L_{2}^{d}[a, b] \\
\stackrel{(32)}{\rightarrow}-L_{1}^{a} L_{1}^{b} L_{2}^{d} c+L_{1}^{a} L_{1}^{c} L_{2}^{d} b .
\end{array}
$$

The latter is trivial modulo (39) and thus reduces to zero by means of (33) or (334) depending on the order on $b, c, d$.

For $n=1$ we have

$$
\begin{aligned}
-L_{1}^{a} L_{1}^{b} R_{1}^{d} c+L_{1}^{a} L_{1}^{c} R_{1}^{d} b+L_{0}^{b} L_{2}^{a} R_{1}^{d} c-L_{0}^{c} L_{2}^{a} R_{1}^{d} b+L_{2}^{a} R_{1}^{d}[c, b] \\
+L_{2}^{b} R_{1}^{d}[c, a]+L_{2}^{c} R_{1}^{d}[a, b] \stackrel{\left(R_{1}\right)}{\rightarrow}-L_{1}^{a} L_{1}^{b} L_{1}^{d} c+L_{1}^{a} L_{1}^{c} L_{1}^{d} b+L_{0}^{b} L_{2}^{a} L_{1}^{d} c \\
\\
\quad-L_{0}^{c} L_{2}^{a} L_{1}^{d} b+L_{2}^{a} L_{1}^{d}[c, b]+L_{2}^{b} L_{1}^{d}[c, a]+L_{2}^{c} L_{1}^{d}[a, b]
\end{aligned}
$$

since the terms containing $e$ annihilate under the action of $L_{n}$. Continue reducing (46) with the rules $(L L)$ and (41):

$$
\begin{array}{r}
-L_{1}^{a} L_{0}^{b} L_{2}^{d} c+L_{1}^{a} L_{0}^{c} L_{2}^{d} b+L_{1}^{a} L_{2}^{d}[b, c]+L_{0}^{b} L_{1}^{d} L_{2}^{a} c+L_{0}^{b} L_{3}^{[a, d]} c-L_{0}^{c} L_{1}^{d} L_{2}^{a} b-L_{0}^{c} L_{3}^{[a, d]} b \\
+L_{1}^{d} L_{2}^{a}[c, b]+L_{3}^{[a, d]}[c, b]+L_{1}^{d} L_{2}^{b}[c, a]+L_{3}^{[b, d]}[c, a]+L_{1}^{d} L_{2}^{c}[a, b]+L_{3}^{[c, d]}[a, b] \\
\stackrel{(L L),\left(L_{3}\right)}{\rightarrow}-L_{0}^{b} L_{1}^{a} L_{2}^{d} c-L_{1}^{[a, b]} L_{2}^{d} c+L_{0}^{c} L_{1}^{a} L_{2}^{d} b+L_{1}^{[a, c]} L_{2}^{d} b+L_{1}^{a} L_{2}^{d}[b, c]+L_{0}^{b} L_{1}^{d} L_{2}^{a} c \\
-L_{0}^{c} L_{1}^{d} L_{2}^{a} b-L_{1}^{d} L_{2}^{a}[b, c]-L_{1}^{d} L_{2}^{b}[a, c]+L_{1}^{d} L_{2}^{c}[a, b] \stackrel{\left(L_{2}\right),(39)}{\rightarrow} 0 .
\end{array}
$$

For $n=0$, first continue reducing (44) and (45) as follows:

$$
L_{1}^{a} L_{1}^{b} R_{0}^{d} c \stackrel{\left(R_{0}\right)}{\rightarrow} L_{1}^{a} L_{1}^{b} L_{0}^{d} c-L_{1}^{a} L_{1}^{b}[d, c] \stackrel{(L L)}{\rightarrow} L_{0}^{d} L_{1}^{a} L_{1}^{b} c+L_{1}^{a} L_{1}^{[b, d]} c+L_{1}^{[a, d]} L_{1}^{b} c-L_{1}^{a} L_{1}^{b}[d, c]
$$




$$
\begin{array}{r}
L_{1}^{a} L_{1}^{c} R_{0}^{d} b+L_{0}^{b} L_{2}^{a} R_{0}^{d} c-L_{0}^{c} L_{2}^{a} R_{0}^{d} b+L_{2}^{a} R_{0}^{d}[c, b]+L_{2}^{b} R_{0}^{d}[c, a]+L_{2}^{c} R_{0}^{d}[a, b] \stackrel{\left(R_{0}\right)}{\rightarrow} L_{1}^{a} L_{1}^{c} L_{0}^{d} b- \\
-L_{1}^{a} L_{1}^{c}[d, b]+L_{0}^{b} L_{2}^{a} L_{0}^{d} c-L_{0}^{b} L_{2}^{a}[d, c]-L_{0}^{c} L_{2}^{a} L_{0}^{d} b+L_{0}^{c} L_{2}^{a}[d, b]+L_{2}^{a} L_{0}^{d}[c, b]-L_{2}^{a}[d,[c, b]] \\
+L_{2}^{b} L_{0}^{d}[c, a]-L_{2}^{b}[d,[c, a]]+L_{2}^{c} L_{0}^{d}[a, b]-L_{2}^{c}[d,[a, b]] \stackrel{(L L)}{\rightarrow} L_{0}^{d} L_{1}^{a} L_{1}^{c} b+L_{1}^{a} L_{1}^{[c, d]} b+L_{1}^{[a, d]} L_{1}^{c} b \\
-L_{1}^{a} L_{1}^{c}[d, b]+L_{0}^{d} L_{0}^{b} L_{2}^{a} c+L_{0}^{[b, d]} L_{2}^{a} c+L_{0}^{b} L_{2}^{[a, d]} c-L_{0}^{b} L_{2}^{a}[d, c]-L_{0}^{d} L_{0}^{c} L_{2}^{a} b-L_{0}^{[c, d]} L_{2}^{a} b-L_{0}^{c} L_{2}^{[a, d]} b \\
+L_{0}^{c} L_{2}^{a}[d, b]+L_{0}^{d} L_{2}^{a}[c, b]+L_{2}^{[a, d]}[c, b]-L_{2}^{a}[d,[c, b]]+L_{0}^{d} L_{2}^{b}[c, a]+L_{2}^{[b, d]}[c, a]-L_{2}^{b}[d,[c, a]] \\
+L_{0}^{d} L_{2}^{c}[a, b]+L_{2}^{[c, d]}[a, b]-L_{2}^{c}[d,[a, b]] .
\end{array}
$$

Now subtract the relations obtained, apply the Jacobi identity to rewrite

$$
\begin{gathered}
L_{2}^{a}[d,[c, b]]=-L_{2}^{a}[c,[b, d]]-L_{2}^{a}[[c, d], b], \quad L_{2}^{b}[d,[c, a]]=-L_{2}^{b}[c,[a, d]]-L_{2}^{b}[[c, d], a], \\
L_{2}^{c}[d,[a, b]]=-L_{2}^{c}[a,[b, d]]-L_{2}^{c}[[a, d], b],
\end{gathered}
$$

and rearrange the summands gathering them into four groups: one starting with $L_{0}^{d}$ and other three containing the Lie brackets $[a, d],[b, d]$, and $[c, d]$ :

$$
\begin{aligned}
& \left(L_{0}^{d} L_{1}^{a} L_{1}^{b} c-L_{0}^{d} L_{1}^{a} L_{1}^{c} b+L_{0}^{d} L_{0}^{c} L_{2}^{a} b-L_{0}^{d} L_{0}^{b} L_{2}^{a} c+L_{0}^{d} L_{2}^{a}[b, c]+L_{0}^{d} L_{2}^{b}[a, c]+L_{0}^{d} L_{2}^{c}[b, a]\right) \\
- & \left(L_{1}^{[a, d]} L_{1}^{c} b-L_{1}^{[a, d]} L_{1}^{b} c+L_{0}^{b} L_{2}^{[a, d]} c-L_{0}^{c} L_{2}^{[a, d]} b+L_{2}^{[a, d]}[c, b]+L_{2}^{b}[c,[a, d]]+L_{2}^{c}[[a, d], b]\right) \\
- & \left(L_{1}^{a} L_{1}^{c}[b, d]-L_{1}^{a} L_{1}^{[b, d]} c+L_{0}^{[b, d]} L_{2}^{a} c-L_{0}^{c} L_{2}^{a}[b, d]+L_{2}^{a}[c,[b, d]]+L_{2}^{[b, d]}[c, a]+L_{2}^{c}[a,[b, d]]\right) \\
- & \left(L_{1}^{a} L_{1}^{[c, d]} b-L_{1}^{a} L_{1}^{b}[c, d]+L_{0}^{b} L_{2}^{a}[c, d]-L_{0}^{[c, d]} L_{2}^{a} b+L_{2}^{a}[[c, d], b]+L_{2}^{b}[[c, d], a]+L_{2}^{[c, d]}[a, b]\right) .
\end{aligned}
$$

The first group is exactly (41) under the action of $L_{0}^{d}$, other three groups coincide with (42). Therefore, the composition reduces to zero by means of (35) and (36).

Observe that the principal parts of the rewriting rules from the Gröbner-Shirshov basis found in Theorem 2 do not depend on the multiplication table of the original Lie algebra $\mathfrak{g}$ as well as on the choice of the form $\langle\cdot \mid \cdot\rangle$. In particular, if $\mathfrak{g}$ is an abelian Lie algebra and $\langle x \mid y\rangle=0$ for all $x, y \in \mathfrak{g}$ then $K(\mathfrak{g})$ is an abelian Lie conformal algebra and $U$ coincides with the 1-dimensional split null extension

$$
0 \rightarrow \mathbb{k} e \rightarrow U \rightarrow \operatorname{Com} \operatorname{Conf}\left(X_{1}, N=3\right) \rightarrow 0
$$

of the free commutative conformal algebra $\operatorname{Com} \operatorname{Conf}\left(X_{1}, N=3\right)$ generated by a linear basis $X_{1}$ of $\mathfrak{g}$ relative to the locality function $N(x, y)=3, x, y \in X_{1}$.

Hence, the linear basis of $\operatorname{Com} \operatorname{Conf}\left(X_{1}, N=3\right)$ consists of all those conformal monomials described in Proposition 1 that are terminal relative to the rewriting rules stated in Theorem 2 .

Corollary 3. Let $Y$ be a linearly ordered set. The linear basis of the free commutative conformal algebra $\operatorname{Com} \operatorname{Conf}(Y, N=3)$ generated by $Y$ relative to the locality 
function $N=3$ consists of the following conformal monomials:

$$
\begin{gathered}
L_{0}^{x_{1}} \ldots L_{0}^{x_{n}} L_{1}^{y_{1}} \ldots L_{1}^{y_{m}} L_{2}^{z} u, \quad x_{i} \leq x_{i+1}, y_{i} \leq y_{i+1} \leq z \leq u, n, m \geq 0, \\
L_{0}^{x_{1}} \ldots L_{0}^{x_{n}} L_{1}^{y_{1}} \ldots L_{1}^{y_{m}} \partial^{s} z, \quad x_{i} \leq x_{i+1}, y_{i} \leq y_{i+1} \leq z, n, m \geq 0, s \geq 1, \\
L_{0}^{x_{1}} \ldots L_{0}^{x_{n}} L_{1}^{y_{1}} \ldots L_{1}^{y_{m}} z, \quad x_{i} \leq x_{i+1}, y_{i} \leq y_{i+1} \leq z, n \geq 0, m=0 \text { or } m \geq 2, \\
L_{0}^{x_{1}} \ldots L_{0}^{x_{n}} L_{1}^{y} z, \quad x_{i} \leq x_{i+1}, x_{n} \leq y \text { or } y \leq z, n \geq 0,
\end{gathered}
$$

where $x_{i}, y_{i}, z, u \in Y$.

If $X=X_{1} \cup\{e\}, Y=X_{1}$ then the above monomials together with the torsion element e form a linear basis of $U=U(K(\mathfrak{g}) ; X, N=3)$.

The conformal algebra $U$ has a natural filtration by degree in $X$. Denote gr $U$ the corresponding associated graded conformal algebra.

Note that every rule in Theorem (2) has the following property: all terms of highest degree in $X$ do not depend on $[\cdot, \cdot]$ and $\langle\cdot \mid \cdot\rangle$. Therefore, if we choose two basic monomials of $U$ described by Corollary 3 and rewrite their conformal product as a linear combination of basic monomials then the terms of highest degree in the expression obtained would be the same as we get for the product of the same monomials in $\operatorname{Com} \operatorname{Conf}\left(X_{1}, N=3\right)$.

As a result, we obtain the following analogue of the Poincaré-Birkhoff-Witt Theorem.

Corollary 4. For every Lie algebra $\mathfrak{g}$ and for every bilinear symmetric invariant form $\langle\cdot, \cdot\rangle$ on $\mathfrak{g}$ we have

$$
\operatorname{gr} U(K(\mathfrak{g}) ; X, N=3) \simeq \operatorname{Com} \operatorname{Conf}\left(X_{1}, N=3\right) \oplus \mathbb{k} e .
$$

Here $X=X_{1} \cup\{e\}$ as above, $X_{1}$ is a basis of $\mathfrak{g}$. In particular,

$$
\operatorname{gr} U\left(\operatorname{Cur} \mathfrak{g} ; X_{1}, N=3\right) \simeq \operatorname{Com} \operatorname{Conf}\left(X_{1}, N=3\right) .
$$

Let us derive another corollary of Theorem 2, Since the case of locality $N=3$ has already been considered we may add a new series of relations to $U(K(\mathfrak{g}) ; X, N=$ $3)$ to get a Gröbner-Shirshov basis of the universal enveloping associative algebra $U(K(\mathfrak{g}) ; X, N=2)$. Namely, it is enough to add relations

$$
L_{2}^{a} b \rightarrow 0, \quad R_{2}^{a} b \rightarrow 0, \quad a, b \in X_{1}
$$

to the Gröbner-Shirshov basis calculated in Theorem 2 and compute all intersections. Since there are no essentially new manipulations, let us just enlist the resulting relations. 
Theorem 3. The set of rewriting rules (28) along with

$$
\begin{gathered}
L_{n}^{a} b \rightarrow 0, \quad n \geq 2, \\
R_{n}^{a} b \rightarrow 0, \quad n \geq 2, \\
R_{1}^{a} b \rightarrow L_{1}^{a} b-\langle a \mid b\rangle e, \\
R_{0}^{a} b \rightarrow L_{0}^{a} b-[a, b], \\
L_{1}^{a} b \rightarrow-L_{1}^{b} a+\langle a \mid b\rangle e, \quad b<a, \\
L_{1}^{a} a \rightarrow \frac{1}{2}\langle a \mid a\rangle e, \\
L_{1}^{a} \partial^{s} b \rightarrow 2 L_{0}^{a} \partial^{s-1} b-L_{0}^{b} \partial^{s-1} a-\partial^{s-1}[a, b], \quad s \geq 1, \\
L_{1}^{a} L_{1}^{b} c \rightarrow 0, \quad a \leq b<c, \\
L_{0}^{a} L_{1}^{b} c \rightarrow L_{0}^{c} L_{1}^{b} a-L_{0}^{b} L_{1}^{c} a+L_{1}^{a}[c, b]+L_{1}^{c}[b, a]+L_{1}^{b}[a, c]+\langle a \mid[b, c]\rangle e \\
b<c<a .
\end{gathered}
$$

is a Gröbner-Shirshov basis of $U=U(K(\mathfrak{g}) ; X, 2)$.

Here the identities (47) -(52) are slightly adjusted (29), (230); and (⒌ as compositions of intersection.

The list of rewriting rules obtained in Theorem 3 has the same properties as in Theorem 2: the principal parts as well as all summands of maximal degree in each rule do not depend neither on a multiplication table on $\mathfrak{g}$ nor on $\langle\cdot, \cdot\rangle$. Hence, the analogue of Corollary 4 holds for $N=2$. In order to complete the description of $U(K(\mathfrak{g}) ; X, N=2)$ we need to state explicitly the set of reduced monomials in the free commutative conformal algebra of locality $N=2$.

Corollary 5. The following monomials form a linear basis of $\operatorname{Com} \operatorname{Conf}(Y, N=2)$, where $Y$ is a linearly ordered set:

$$
\begin{gathered}
L_{0}^{x_{1}} \ldots L_{0}^{x_{n}} \partial^{s} z, \quad x_{1} \leq \cdots \leq x_{n}, \quad s \geq 0, \\
L_{0}^{x_{1}} \ldots L_{0}^{x_{n}} L_{1}^{y} z, \quad x_{1} \leq \cdots \leq x_{n} \leq z, y<z,
\end{gathered}
$$

for $x_{i}, y, z \in Y$.

\section{REFERENCES}

[1] A. A. Balinskii, S. P. Novikov, Poisson brackets of hydrodynamic type, Frobenius algebras and Lie algebras, Sov. Math. Dokl. 32 (1985), 228-231.

[2] B. Bakalov, A. D'Andrea, V. G. Kac, Theory of finite pseudoalgebras, Adv. Math. 162 (2001) $1-140$.

[3] B. Bakalov, V. G. Kac, A. Voronov, Cohomology of conformal algebras, Comm. Math. Phys. 200 (1999) 561-589.

[4] A. A. Beilinson, V. G. Drinfeld, Chiral algebras, Amer. Math. Soc. Colloquium Publications 51 (AMS, Providence, RI, 2004).

[5] A. A. Belavin, A. M. Polyakov, A. B. Zamolodchikov, Infinite conformal symmetry in twodimensional quantum field theory, Nuclear Phys. 241 (1984), 333-380.

[6] G. M. Bergman, The diamond lemma for ring theory, Adv. Math. 29 (1978), 178-218. 
[7] L. A. Bokut, Imbeddings into simple associative algebras (Russian), Algebra i Logika 15 (1976), $117-142$.

[8] L. A. Bokut, Y. Fong, W.-F. Ke, Composition-diamond lemma for associative conformal algebras, J. Algebra 272 (2004) 739-774.

[9] L. A. Bokut, Y.-Q. Chen, Gröbner-Shirshov bases and their calculation, Bull. Math. Sci. 4 (2014), 325-395.

[10] M. R. Bremner, V. V. Dotsenko, Algebraic operads. An algorithmic companion, CRC Press, Boca Raton, FL (2016).

[11] S.-J. Cheng, V. G. Kac, Conformal modules, Asian J. Math. 1 (1997) 181-193.

[12] E. Frenkel, D. Ben-Zvi, Vertex algebras and algebraic curves, Mathematical Surveys and Monograps, vol. 88. American Mathematical Society, Providence (2001).

[13] I. M. Gelfand, I. Ya. Dorfman, Hamilton operators and associated algebraic structures, Funct. Anal. Appl. 13 (1979) no. 4, 13-30.

[14] M. Golenishcheva-Kutuzova, V. G. Kac, Г-conformal algebras, J. Math. Phys. 39 (1998) 2290-2305.

[15] Y. Hong, Z. Wu, Simplicity of quadratic Lie conformal algebras, Comm. Algebra 45 (2017) (1) $141-150$.

[16] V. G. Kac, Vertex algebras for beginners (Second ed.), University Lecture Series 10, American Mathematical Society, Providence (1998).

[17] S.-J. Kang, K.-H. Lee, Gröbner-Shirshov bases for representation theory, J. Korean Math. Soc. 37 (2000) 55-72.

[18] P. S. Kolesnikov, Conformal algebras in the context of linear algebraic groups, in: Generalized Lie Theory in Mathematics, Physics and Beyond (S. Silvestrov et al. eds), Springer Verl., Berlin, Heidelberg (2009) 235-246.

[19] P. S. Kolesnikov, Graded associative conformal algebras of finite type, Algebras and Repr. Theory vol. 16, 62013 1521-1539.

[20] P. S. Kolesnikov, Gröbner-Shirshov bases for associative conformal algebras with arbitrary locality function, New Trends in Algebra and Combinatorics, Proceedings of the 3rd International Congress in Algebra and Combinatorics (K. P. Shum et al, Eds.), World Scientific (2020), 255-267.

[21] P. S. Kolesnikov, Universal enveloping Poisson conformal algebras, Int. J. of Alg. and Comp. 30 (2020) no 5, 1015-1034.

[22] P. S. Kolesnikov, R. A. Kozlov, A. S. Panasenko, Quadratic Lie conformal superalgebras related to Novikov superalgebras, arXiv:1912.03943v1.

[23] M. H. A. Newman, On theories with a combinatorial definition of 'equivalence'. Ann. Math. 43, (1942) 223-243.

[24] L. Ni, Y.-Q. Chen, A new Composition-Diamond lemma for associative conformal algebras, J. Algebra App. 16 (2017), 1750094-1-1750094-28.

[25] M. Roitman, On free conformal and vertex algebras, J. Algebra 217, (1999) 496-527.

[26] M. Roitman, Universal enveloping conformal algebras, Sel. Math., New Ser. 6 (2000) 319-345.

[27] X. Xu, Quadratic conformal superalgebras, J. Algebra 231 (2000), 1-38.

1) Sobolev Institute of Mathematics, Novosibirsk, Russia

2) Novosibirsk State University, Novosibirsk, Russia 